\title{
Intrinsic double-peak structure of the specific heat in low-dimensional quantum ferrimagnets
}

\author{
Takashi Nakanishi and Shoji Yamamoto \\ Department of Physics, Okayama University, Tsushima, Okayama 700-8530, Japan
}

(18 February 2002)

\begin{abstract}
Motivated by recent magnetic measurements on $A_{3} \mathrm{Cu}_{3}\left(\mathrm{PO}_{4}\right)_{4}(A=\mathrm{Ca}, \mathrm{Sr})$ and $\mathrm{Cu}(3 \text {-Clpy })_{2}\left(\mathrm{~N}_{3}\right)_{2}$ (3-Clpy $=3$-Chloropyridine), both of which behave like one-dimensional ferrimagnets, we extensively investigate the ferrimagnetic specific heat with particular emphasis on its double-peak structure. Developing a modified spin-wave theory, we reveal that ferromagnetic and antiferromagnetic dual features of ferrimagnets may potentially induce an extra low-temperature peak as well as a Schottky-type peak at mid temperatures in the specific heat.
\end{abstract}

PACS numbers: 75.10.Jm, 75.40.Cx, 75.30.Ds, 75.40.Mg

Recent progress on the theoretical understanding of low-dimensional (low-D) quantum ferrimagnets deserves special mention in their long history of research. A minimum of the susceptibility $(\chi)$-temperature $(T)$ product has been known as typical of 1-D ferrimagnets [1]. Although the $T^{-1}$-diverging $\chi T$ at low temperatures is reminiscent of the ferromagnetic susceptibility, it turns into the high-temperature paramagnetic behavior showing the antiferromagnetic increase. Recently an explicit sum rule [2] for the ferrimagnetic susceptibility has been found: Spin- $(S, s)$ ferrimagnetic chains behave similar to combinations of spin- $(S-s)$ ferromagnetic and spin- $(2 s)$ antiferromagnetic chains provided $S=2 s$. An epochal argument [3] on the ground-state magnetization curves of low-D quantum magnets stimulated broad interest in ferrimagnetic chains [4,5] and ladders [6] in a field. Spin- $(S, s)$ ferrimagnetic chains were found to exhibit $2 s$ magnetization plateaus without any bond alternation [7. The discovery of a metal-oxide Haldane-gap antiferromagnet $\mathrm{Y}_{2} \mathrm{BaNiO}_{5}$ [8,9] and its rare-earth derivatives $R_{2} \mathrm{BaNiO}_{5}$ [10,11] directed our attention to 2-D mixedspin magnets. Their magnetic double structure, that is, the coexistence of gapless and gapped excitations, was well interpreted from the point of view of coupled ferrimagnetic chains [12]. Nuclear-magnetic-resonance measurements 13 on an ordered bimetallic chain compound $\mathrm{NiCu}\left(\mathrm{C}_{7} \mathrm{H}_{6} \mathrm{~N}_{2} \mathrm{O}_{6}\right)\left(\mathrm{H}_{2} \mathrm{O}\right)_{3} \cdot 2 \mathrm{H}_{2} \mathrm{O}$ revealed a unique field dependence of the relaxation rate, $T_{1}^{-1} \propto H^{-1 / 2}$, which was found to be indirect observation of ferrimagnetic dispersion relations [14.

In this article, we discuss another hot topic on the ferrimagnetic specific heat $(C)$. Intertwining doublechain ferrimagnets $A_{3} \mathrm{Cu}_{3}\left(\mathrm{PO}_{4}\right)_{4}(A=\mathrm{Ca}, \mathrm{Sr})$ 15 and a ferromagnetic-ferromagnetic-antiferromagneticantiferromagnetic bond-alternating chain ferrimagnet $\mathrm{Cu}(3 \text {-Clpy })_{2}\left(\mathrm{~N}_{3}\right)_{2}$ (3-Clpy = 3-Chloropyridine) [16, which are illustrated in Figs. 11(b) and 1 (c), respectively, were both reported to exhibit a unique temperature dependence of the specific heat: A minimum at low temperatures and then a noticeable increase toward a Schottkytype maximum. Ferrimagnets generally possess a ground state of macroscopically degenerate multiplet and there- fore an applied field may induce a double-peaked specific heat [17]. However, such an extrinsic mechanism should be distinguished from the intrinsic thermodynamics. Magnetic measurements [15, 18, 19 were also performed for these materials in an attempt to evaluate the exchange couplings but no parametrization reported so far is so consistent as to fit the magnetization, susceptibility, and specific heat consistently. Thus motivated, we calculate the specific heat for a wide class of 1-D ferrimagnets and reveal its intrinsic and generic features.

(a)

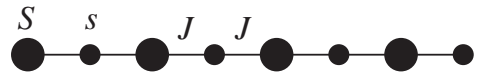

(b)

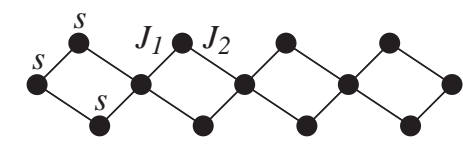

(c)

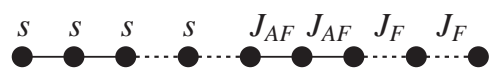

FIG. 1. Schematic representations of the bimetallic chain compounds (a), the trimeric intertwining double-chain compounds (b), and the tetrameric bond-alternating chain compounds (c), which are described by the Hamiltonians (1a), (1b), and (1c), respectively, where smaller and larger bullet symbols denote spins $s=\frac{1}{2}$ and $S>\frac{1}{2}$, while solid and dashed segments mean antiferromagnetic and ferromagnetic exchange couplings between them, respectively.

The simplest quantum ferrimagnet in one dimension consists of two kinds of spins $S$ and $s$ alternating on a ring with antiferromagnetic exchange coupling between nearest neighbors and a series of such family compounds were extensively synthesized by Verdaguer, Kahn, and their coworkers [1]. One of their works is bimetallic chains of general formula $A \mathrm{Cu}(\mathrm{pbaOH})\left(\mathrm{H}_{2} \mathrm{O}\right)_{3} \cdot n \mathrm{H}_{2} \mathrm{O}(A=\mathrm{Ni}, \mathrm{Co}, \mathrm{Fe}, \mathrm{Mn}$; pbaOH $=2$-hydroxy-1,3-propylenebis(oxamato) [20], which are illustrated in Fig. 1(a). We first consider these bimetallic chain compounds in order to verify the validity of our method, a modified spin-wave theory, as well as to understand typical features of 1-D ferrimagnets, and then proceed to the above-mentioned trimeric cop- 
per phosphates and tetrameric copper complex, whose Hamiltonians can be written as

$$
\begin{gathered}
\mathcal{H}=J \sum_{n}\left(\boldsymbol{S}_{n} \cdot \boldsymbol{s}_{n}+\boldsymbol{s}_{n} \cdot \boldsymbol{S}_{n+1}\right) \\
\mathcal{H}=\sum_{n}\left[J_{1}\left(\boldsymbol{s}_{n, 1} \cdot \boldsymbol{s}_{n, 2}+\boldsymbol{s}_{n, 2} \cdot \boldsymbol{s}_{n, 3}\right)\right. \\
\left.\quad+J_{2}\left(\boldsymbol{s}_{n, 2} \cdot \boldsymbol{s}_{n+1,1}+\boldsymbol{s}_{n, 3} \cdot \boldsymbol{s}_{n+1,2}\right)\right] \\
\mathcal{H}=\sum_{n}\left[J_{\mathrm{AF}}\left(\boldsymbol{s}_{n, 1} \cdot \boldsymbol{s}_{n, 2}+\boldsymbol{s}_{n, 2} \cdot \boldsymbol{s}_{n, 3}\right)\right. \\
\left.\quad-J_{\mathrm{F}}\left(\boldsymbol{s}_{n, 3} \cdot \boldsymbol{s}_{n, 4}+\boldsymbol{s}_{n, 4} \cdot \boldsymbol{s}_{n+1,1}\right)\right]
\end{gathered}
$$

respectively. In the following, we take $N$ as the number of unit cells and set the length of the unit cell to unity.

Assuming the Néel-like order and introducing the bosonic operators for the spin deviation in each sublattice, we can expand the Hamiltonian (1a) as

$$
\mathcal{H}=E_{\text {class }}+\mathcal{H}_{0}+\mathcal{H}_{1}+O\left(S^{-1}\right),
$$

where $E_{\text {class }}=-2 S s J N$ is the classical ground-state energy, $\mathcal{H}_{0}$ gives the free spin waves, and $\mathcal{H}_{1}$ describes twobody interactions between them, which are, respectively, the $O\left(S^{2}\right), O\left(S^{1}\right)$, and $O\left(S^{0}\right)$ terms. It may be an idea to diagonalize $\mathcal{H}_{0}$ and $\mathcal{H}_{1}$ simultaneously. However, the resultant dispersion relations are gapped and thus misread the low-temperature ferromagnetic features [21] inherent in 1-D ferrimagnets, such as the $T^{1 / 2}$-vanishing $C$ and the $T^{-2}$-diverging $\chi$. Hence we propose another treatment 22] of the up-to- $O\left(S^{0}\right)$ bosonic Hamiltonian. $\mathcal{H}_{0}$ is diagonalized as

$$
\mathcal{H}_{0}=E_{0}+J \sum_{k}\left(\omega_{k}^{-} \alpha_{k}^{\dagger} \alpha_{k}+\omega_{k}^{+} \beta_{k}^{\dagger} \beta_{k}\right),
$$

where $E_{0}=J \sum_{k}\left[\omega_{k}-(S+s)\right]$ is the $O\left(S^{1}\right)$ quantum correction to the ground-state energy, and $\alpha_{k}^{\dagger}$ and $\beta_{k}^{\dagger}$ are the creation operators of the ferromagnetic and antiferromagnetic spin waves of momentum $k$ whose dispersion relations are given by $\omega_{k}^{ \pm}=\omega_{k} \pm(S-s)$ with $\omega_{k}=\left[(S-s)^{2}+4 S s \sin ^{2}(k / 2)\right]^{1 / 2}$. Using the Wick theorem, $\mathcal{H}_{1}$ is rewritten as

$$
\begin{aligned}
\mathcal{H}_{1}= & E_{1}-J \sum_{k}\left(\delta \omega_{k}^{-} \alpha_{k}^{\dagger} \alpha_{k}+\delta \omega_{k}^{+} \beta_{k}^{\dagger} \beta_{k}\right) \\
& +\mathcal{H}_{\text {irrel }}+\mathcal{H}_{\text {resid }},
\end{aligned}
$$

where the $O\left(S^{0}\right)$ correction to the ground-state energy and those to the dispersions are given by $E_{1}=-2 J N\left[\Gamma_{1}^{2}+\Gamma_{2}^{2}+(\sqrt{S / s}+\sqrt{s / S}) \Gamma_{1} \Gamma_{2}\right]$ and $\delta \omega_{k}^{ \pm}=2(S+s)\left(\Gamma_{1} / \omega_{k}\right) \sin ^{2}(k / 2)+\left(\Gamma_{2} / \sqrt{S s}\right)\left[\omega_{k} \pm\right.$ $\left.\left(S^{-}-s\right)\right]$ with $\Gamma_{1}=(2 N)^{-1} \sum_{k}\left[(S+s) / \omega_{k}-\right.$ 1] and $\Gamma_{2}=-N^{-1} \sum_{k}\left(\sqrt{S s} / \omega_{k}\right) \cos ^{2}(k / 2)$, while the irrelevant one-body terms $\mathcal{H}_{\text {irrel }}=-J(S-$ $s)^{2}\left(\Gamma_{1} / \sqrt{S s}\right) \sum_{k}\left[\cos (k / 2) / \omega_{k}\right]\left(\alpha_{k} \beta_{k}+\alpha_{k}^{\dagger} \beta_{k}^{\dagger}\right)$ and the residual two-body interactions $\mathcal{H}_{\text {resid }}$ are both neglected so as to keep the ferromagnetic branch gapless. This procedure may be recognized as the perturbational treatment of $\mathcal{H}_{1}$ to $\mathcal{H}_{0}$. The resultant Hamiltonian is compactly represented as

$$
\mathcal{H} \simeq E_{\mathrm{g}}+J \sum_{k}\left(\widetilde{\omega}_{k}^{-} \alpha_{k}^{\dagger} \alpha_{k}+\widetilde{\omega}_{k}^{+} \beta_{k}^{\dagger} \beta_{k}\right),
$$

with $\widetilde{\omega}_{k}^{ \pm}=\omega_{k}^{ \pm}-\delta \omega_{k}^{ \pm}$and $E_{\mathrm{g}}=E_{\text {class }}+E_{0}+E_{1}$.

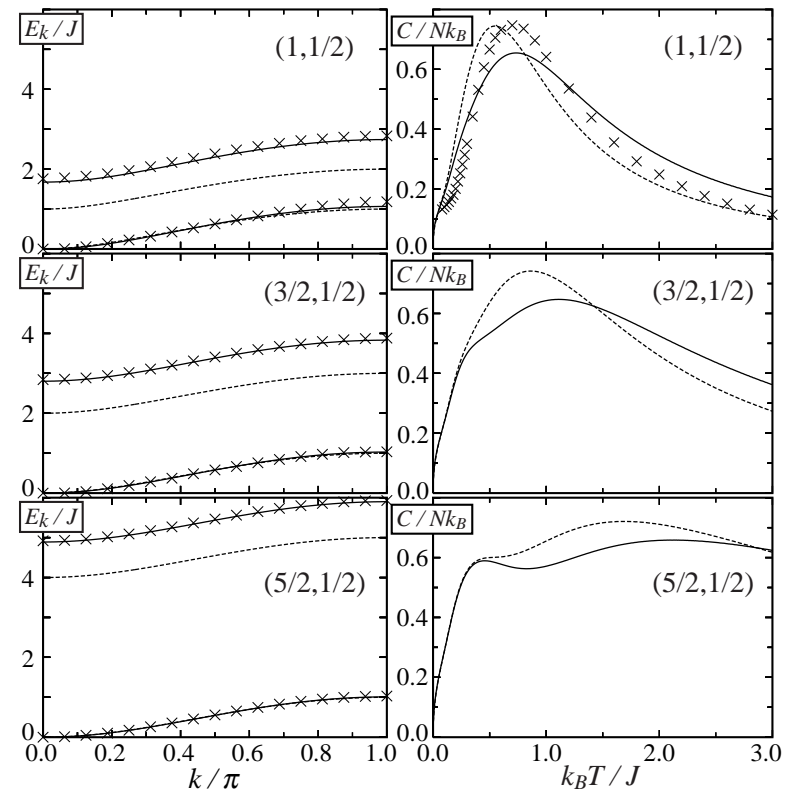

FIG. 2. Noninteracting (dashed lines) and interacting (solid lines) spin-wave calculations of the dispersion relations and the specific heat for the alternating-spin chains which are described by the Hamiltonian (1a) and Fig. 1(a). Corresponding quantum Monte Carlo calculations $(\times)$ are also shown for comparison.

In Fig. 2, the dispersion relations up to the order $O\left(S^{1}\right)$ and $O\left(S^{0}\right), \omega_{k}^{ \pm}$and $\widetilde{\omega}_{k}^{ \pm}$, are compared with the numerical findings obtained through a recently developed quantum Monte Carlo scheme [23]. We find two distinct branches of spin-wave excitations. The gapless one is made of the elementary excitations reducing the groundstate magnetization and is thus of ferromagnetic aspect, while the gapped one, enhancing the ground-state magnetization, is of antiferromagnetic aspect. The ferromagnetic spin waves indeed exhibit a quadratic dispersion at small momenta. The $O\left(S^{0}\right)$ quantum correction has a significant effect on the antiferromagnetic spin waves, whereas the ferromagnetic ones look almost free from interaction.

The core idea 24] of the so-called modified spin-wave theory can be summarized as reliably describing thermodynamics even in low dimensions by introducing a constraint on the magnetization. We demonstrate its ferrimagnetic version [25] for the alternating-spin chains. Constraining the total magnetization to be zero, Taka- 
hashi [26] obtained an excellent description of the lowtemperature thermodynamics of 1- and 2-D Heisenberg ferromagnets. His idea that the thermal spin deviation should be equal to the ground-state magnetization may be replaced by

$$
(S+s) N=(S+s) \sum_{k}\left(n_{k}^{-}+n_{k}^{+}\right) / \omega_{k},
$$

for our ferrimagnets, where $n_{k}^{ \pm}=\sum_{n^{-}, n^{+}} n^{ \pm} P_{k}\left(n^{-}, n^{+}\right)$ with $P_{k}\left(n^{-}, n^{+}\right)$being the probability of $n^{-}$ferromagnetic and $n^{+}$antiferromagnetic spin waves appearing in the $k$-momentum state. Equation (6) claims that the thermal fluctuation should cancel the Néel-state staggered magnetization instead of the uniform one, in response to the replacement of the ferromagnetic exchange coupling by the antiferromagnetic one. Minimizing the free energy

$$
\begin{aligned}
F= & E_{\mathrm{g}}+\sum_{k} \sum_{\sigma= \pm} n_{k}^{\sigma} \widetilde{\omega}_{k}^{\sigma} \\
& +k_{\mathrm{B}} T \sum_{k} \sum_{n^{-}, n^{+}} P_{k}\left(n^{-}, n^{+}\right) \ln P_{k}\left(n^{-}, n^{+}\right),
\end{aligned}
$$

with respect to $P_{k}$ at each $k$ under the condition (5) as well as the trivial constraints $\sum_{n^{-}, n^{+}} P_{k}\left(n^{-}, n^{+}\right)=1$, we obtain the free and internal energies at thermal equilibrium as $F=E_{\mathrm{g}}+\mu(S-s) N-k_{\mathrm{B}} T \sum_{k} \sum_{\sigma= \pm} \ln \left(1+\bar{n}_{k}^{\sigma}\right)$ and $U=E_{\mathrm{g}}+J \sum_{k} \sum_{\sigma= \pm} \bar{n}_{k}^{\sigma} \widetilde{\omega}_{k}^{\sigma}$, where the optimum distributions are given by $\bar{n}_{k}^{ \pm}=\left\{\mathrm{e}^{\left[{ }^{\left[J \widetilde{\omega}_{k}^{ \pm}-\mu(S+s) / \omega_{k}\right] / k_{\mathrm{B}} T}-\right.}\right.$ $1\}^{-1}$ with a Lagrange multiplier $\mu$ obtained through Eq. (6). The thus-obtained specific heat, together with its interaction-free version, is shown in Fig. 2. In the case of $(S, s)=\left(1, \frac{1}{2}\right)$, we compare the present results with the quantum Monte Carlo estimates. Considering that the conventional antiferromagnetic spin-wave theory does not work at all for the 1-D thermodynamics, the present calculations surprisingly well describe the overall temperature dependence, including the $\sqrt{T}$ initial behavior and the Schottky-type peak. When we employ the interacting spin waves, the most significant improvement is the correction of the peak position. Although they somewhat underestimate the peak height, the Schottky-type anomaly is then correctly located, which is essential to reproduce the overall structure. The specific heat may be double-peaked provided the antiferromagnetic gap is much larger than the ferromagnetic band width, which is expressed in terms of the interacting spin waves as $\widetilde{\omega}_{k=0}^{+} \gg \widetilde{\omega}_{k=\pi}^{-}-\widetilde{\omega}_{k=0}^{-}$. This condition is satisfied for $S \gg 2 s$ and the ferrimagnetic chain of $(S, s)=\left(\frac{5}{2}, \frac{1}{2}\right)$ indeed exhibits a double-peaked specific heat. In the following, we argue the other mechanism for a varied temperature dependence, which is of topological origin and may thus be valid for homometallic 1-D ferrimagnets. Considering the poor convergence of quantum Monte Carlo calculations at low temperatures, the present modified spin-wave scheme is one of the most reliable and feasible approach.

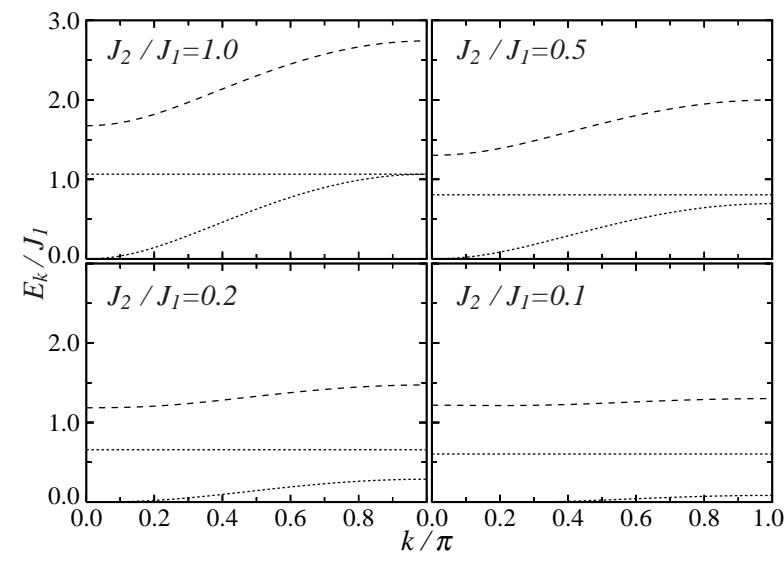

FIG. 3. Interacting spin-wave calculations of the dispersion relations for the trimeric chains which are described by the Hamiltonian (1b) and Fig. 1(b), where the ferromagnetic and antiferromagnetic excitations are distinguishably shown by dotted and dashed lines, respectively.

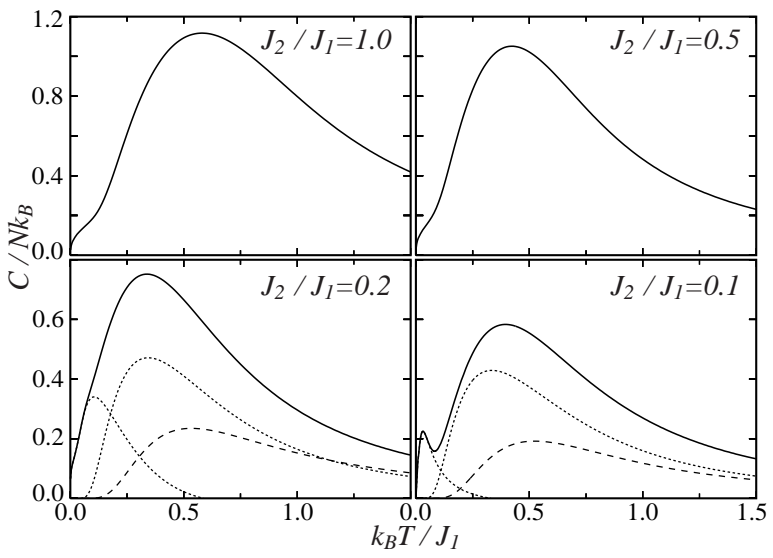

FIG. 4. Interacting spin-wave calculations of the specific heat for the trimeric chains which are described by the Hamiltonian (1b) and Fig. 1(b), where individual contributions of the ferromagnetic and antiferromagnetic spin waves are also shown by dotted and dashed lines, respectively, the sum of which is equal to the total drawn by solid lines.

The dispersion relations and the resultant specific heat of the trimeric intertwining chains are shown in Figs. 3 and 4 , respectively. The system is analogous to the alternating-spin chain of $(S, s)=\left(1, \frac{1}{2}\right)$ but displays an additional flat band which is gapped but of ferromagnetic aspect. With decreasing $J_{2}$ in comparison with $J_{1}$, a second anomaly appears at low temperatures. The present tool advantageously enables us to observe each contribution of the distinct excitation bands. We find that due to the existence of the mid band, the double-peak structure is limited to rather small ratios $J_{2} / J_{1}$. In the previous experiments [15], the minimum related to the second anomaly was indeed observed, but the measured temperatures $(1.8 \sim 15 \mathrm{~K})$ were not low enough to estimate the maximum of the bump. A significant increase and the following Schottky-type main peak at higher temperatures 
were not explicitly shown either, where the magnetic and lattice contributions should carefully be separated. Our calculations fully motivate further measurements [18] and make possible detailed analyses of them.

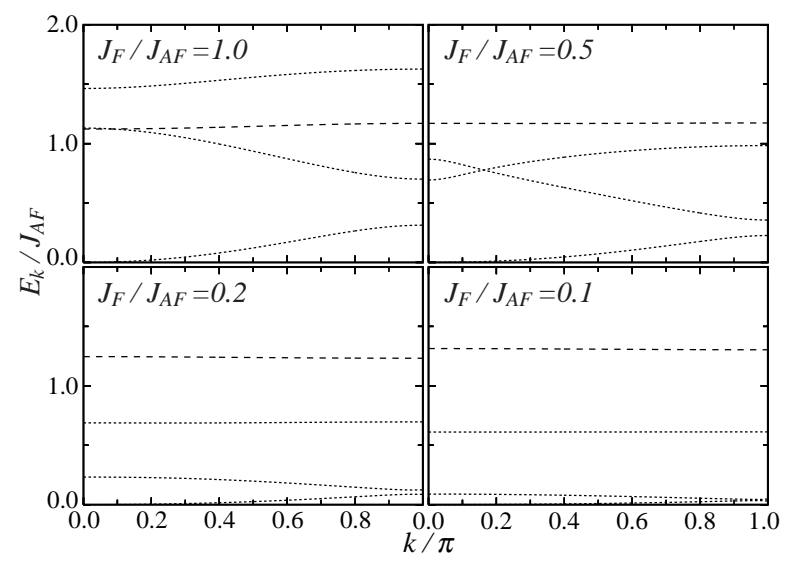

FIG. 5. Interacting spin-wave calculations of the dispersion relations for the tetrameric chains which are described by the Hamiltonian (1c) and Fig. 1(c), where the ferromagnetic and antiferromagnetic excitations are distinguishably shown by dotted and dashed lines, respectively.

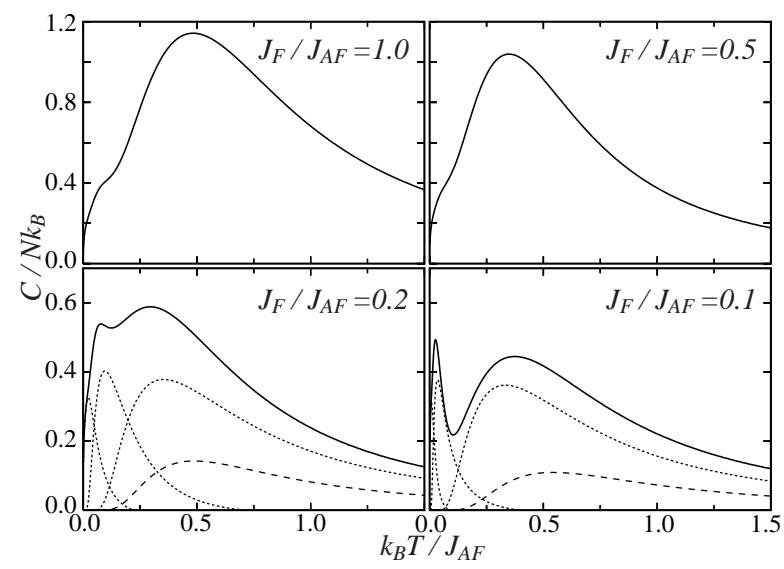

FIG. 6. Interacting spin-wave calculations of the specific heat for the tetrameric chains which are described by the Hamiltonian (1c) and Fig. 1(c), where individual contributions of the ferromagnetic and antiferromagnetic spin waves are also shown by dotted and dashed lines, respectively, the sum of which is equal to the total drawn by solid lines.

The tetrameric bond-alternating chains are discussed in Figs. 5 and 6. We again find a double-peaked specific heat of topological origin. The lower two bands construct the low-temperature bump, while the upper two contribute to the main peak at mid temperatures. It is the double contribution that makes the second peak much more noticeable in the tetrameric chains than in the trimeric chains. The susceptibility measurements [19,27 were well interpreted by setting $J_{\mathrm{F}} / J_{\mathrm{AF}}$ to 0.5 . However, such parameters neither induce any detectable second peak of the specific heat within the Hamiltonian
(10) nor fit the recent observations [16] detecting the second anomaly around $0.5 \mathrm{~K}$. We have tried the opposite parametrizations $J_{\mathrm{AF}}<J_{\mathrm{F}}$. However, the third band lying much closer to the lower two makes the lowtemperature anomaly much more conspicuous and suppress the Schottky-type peak, ending up with complete discrepancy with the observations. Further measurements and more extensive analyses, for instance, taking account of exchange anisotropy, are necessary for the total understanding.

The trimeric and tetrameric chain materials have been measured by separate groups under their respective motivations. Interestingly, however, the unique temperature dependences of their specific heat potentially originate in the same mechanism. We have revealed the intrinsic double-peak structure of the specific heat in low-D ferrimagnets of topological origin. From this point of view, homometallic or organic ferrimagnets should further be synthesized and measured, where the double-peaked specific heat may generically be observed.

This work was supported by the Japanese Ministry of Education, Science, and Culture and by the Sumitomo Foundation. The numerical calculation was done using the facility of the Supercomputer Center, Institute for Solid State Physics, University of Tokyo.

[1] O. Kahn, Struct. Bonding (Berlin) 68, 89 (1987); O. Kahn, Y. Pei, and Y. Journaux, in Inorganic Materials, edited by D. W. Bruce and D. O'Hare (Wiley, New York, 1995), p. 95.

[2] S. Yamamoto, Phys. Rev. B 59, 1024 (1999); S. Yamamoto, T. Fukui, and T. Sakai, Eur. Phys. J. B. 15 (2000) 211.

[3] M. Oshikawa, M. Yamanaka, and I. Affleck, Phys. Rev. Lett. 78, 1984 (1997).

[4] T. Kuramoto, J. Phys. Soc. Jpn. 67, 1762 (1998); 68, 1813 (1999).

[5] T. Sakai and S. Yamamoto, Phys. Rev. B 60, 4053 (1999); J. Phys.: Condens. Matter 12, 9787 (2000).

[6] A. Langari and M. A. Martin-Delgado, Phys. Rev. B 62, 11725 (2000).

[7] S. Yamamoto and T. Sakai, Phys. Rev. 62, 3795 (2000).

[8] J. Darriet and L. P. Regnault, Solid State Commun. 86, 409 (1993).

[9] J. F. DiTusa, S.-W. Cheong, J.-H. Park, G. Aeppli, C. Broholm, and C. T. Chen, Phys. Rev. Lett. 73, 1857 (1994).

[10] A. Zheludev, J. M. Tranquada, T. Vogt, and D. J. Buttrey, Phys. Rev. 54, 6437 (1996); 54, 7210 (1996).

[11] T. Yokoo, A. Zheludev, M. Nakamura, and J. Akimitsu, Phys. Rev. 55, 11516 (1997); T. Yokoo, S. Raymond, A. Zheludev, S. Maslov, E. Ressouche, I. Zaliznyak, R. Erwin, M. Nakamura, and J. Akimitsu, ibid., 58, 14424 (1998). 
[12] Y. Takushima, A. Koga, and N. Kawakami, Phys. Rev. 61, 15189 (2000).

[13] N. Fujiwara and M. Hagiwara, Solid State Commun. 113, $433(2000)$.

[14] S. Yamamoto, Phys. Rev. 61, R842 (2000); Phys. Lett. A 265, 139 (2000); J. Phys. Soc. Jpn. 69, 2324 (2000).

[15] M. Drillon, M. Belaiche, P. Legoll, J. Aride, A. Boukhari, and A. Moqine, J. Magn. Magn. Mater. 128, 83 (1993).

[16] M. Hagiwara and H. Katori, private communication.

[17] K. Maisinger, U. Schollwöck, S. Brehmer, H.-J. Mikeska, and S. Yamamoto, Phys. Rev. B 58, R5908 (1998).

[18] Y. Ajiro, T. Asano, K. Nakaya, M. Mekata, K. Ohyama, Y. Yamaguchi, Y. Koike, Y. Morii, K. Kamishima, H. Katori, and T. Goto, J. Phys. Soc. Jpn. Suppl. A 70, 186 (2001).

[19] M. Hagiwara, Y. Narumi, K. Minami, and K. Kindo, Physica B 294-295, 30 (2001).

[20] O. Kahn, Y. Pei, M. Verdaguer, J.-P. Renard, and J.
Sletten, J. Am. Chem. Soc. 110, 782 (1988); P. J. van Koningsbruggen, O. Kahn, K. Nakatani, Y. Pei, J.-P. Renard, M. Drillon, and P. Legoll, Inorg. Chem. 29, 3325 (1990).

[21] S. Brehmer, H.-J. Mikeska, and S. Yamamoto, J. Phys.: Condens. Matter 9, 3921 (1997); S. Yamamoto, S. Brehmer, and H.-J. Mikeska, Phys. Rev. B 57, 13610 (1998).

[22] S. Yamamoto, T. Fukui, K. Maisinger, and U. Schollwöck, J. Phys.: Condens. Matter 10, 11033 (1998).

[23] S. Yamamoto, Phys. Rev. Lett. 75, 3348 (1995); S. Yamamoto and S. Miyasita, Phys. Lett. A 235, 545 (1997).

[24] M. Takahashi, Prog. Theor. Phys. Suppl. 87, 233 (1986).

[25] S. Yamamoto and T. Fukui, Phys. Rev. B 57, 14008 (1998).

[26] M. Takahashi, Phys. Rev. Lett. 58, 168 (1987).

[27] A. Escuer, R. Vicente, M. S. E. Fallah, M. A. S. Goher, and F. A. Mautner, Inorg. Chem. 37, 4466 (1998). 